\title{
LOWER BOUNDS ON THE HAUSDORFF MEASURE OF NODAL SETS
}

\author{
Christopher D. Sogge And Steve Zelditch
}

AbStract. Let $\mathcal{N}_{\varphi_{\lambda}}$ be the nodal hypersurface of a $\Delta$-eigenfunction $\varphi_{\lambda}$ of eigenvalue $\lambda^{2}$ on a smooth Riemannian manifold. We prove that $\mathcal{H}^{n-1}\left(\mathcal{N}_{\varphi_{\lambda}}\right) \geq C \lambda^{\frac{7}{4}-\frac{3 n}{4}}$. The best previous lower bound was $e^{-C \lambda}$.

Let $(M, g)$ be a compact $C^{\infty}$ Riemannian manifold of dimesion $n$, let $\varphi_{\lambda}$ be an $L^{2}$-normalized eigenfunction of the Laplacian,

$$
\Delta \varphi_{\lambda}=-\lambda^{2} \varphi_{\lambda}
$$

and let

$$
\mathcal{N}_{\varphi_{\lambda}}=\left\{x: \varphi_{\lambda}(x)=0\right\}
$$

be its nodal hypersurface. Let $\mathcal{H}^{n-1}\left(\mathcal{N}_{\varphi_{\lambda}}\right)$ denote its $(n-1)$-dimensional Riemannian hypersurface measure. In this note we prove

TheOREM 1. For any $C^{\infty}$ metric $g$, there exists a constant $C_{g}>0$ so that

$$
\mathcal{H}^{n-1}\left(\mathcal{N}_{\varphi_{\lambda}}\right) \geq C_{g} \lambda^{\frac{7}{4}-\frac{3 n}{4}} .
$$

The proof of Theorem 1 is based on a special case of a general identity (Proposition 1 ) which has other interesting implications. In $\S 3$ we consider possible improvements that might be derived from other cases of the identity in Proposition 1.

Some background on lower bounds on volumes of nodal hypersurfaces: In [Y], S. T. Yau conjectured that for any $C^{\infty}$ metric, one should have

$$
c \lambda \leq \mathcal{H}^{n-1}\left(\mathcal{N}_{\varphi_{\lambda}}\right) \leq C \lambda .
$$

Here, and elsewhere in this article, $C, c$ denote some positive constants depending only on $(M, g)$ and not on $\lambda$. Both the upper and lower bounds were proved for real analytic $C^{\omega}$ metrics by Donnelly-Fefferman in [DF]. However, for $C^{\infty}$ metrics the best previous result appears to be

$$
C^{-\lambda} \leq \mathcal{H}^{n-1}\left(\mathcal{N}_{\varphi_{\lambda}}\right) \leq \lambda^{C \lambda} .
$$

The upper bound was first proved in [HS] and the lower bound is proved in Theorem 6.2 .5 of [HL]. In dimension 2, J. Brüning proved the lower bound of (1) (see also [Sa]). Thus, our lower bound appears to be the first one that breaks the exponential barrier in dimensions $n \geq 3$. Perhaps surprisingly, the proof is quite simple.

In dimension 2, Donnelly-Fefferman [DF2] and Dong [D] proved the upper bound $\mathcal{H}^{1}\left(\mathcal{N}_{\varphi_{\lambda}}\right) \leq C \lambda^{3 / 2}$ when $\operatorname{dim} M=2$. In dimensions $n \geq 3$, the Hardt-Simon $\lambda^{\lambda}$ upper bound in (2) still seems to be the only known bound. The approach taken in this

Received by the editors September 18, 2010.

Research partially supported by NSF grants, DMS-904839 and DMS-0904252. 
note might lead to improvements in the upper bound, but not as simply as for the lower bound.

The proof of Theorem 1 is based on the following identity, which was inspired by a closely related identity of $\mathrm{R}$. T. Dong $[\mathrm{D}]$ ( see also $[\mathrm{ACF}]$ ).

Proposition 1. For any $C^{\infty}$ Riemannian manifold, we have,

$$
2 \int_{\mathcal{N}_{\varphi_{\lambda}}}\left|\nabla \varphi_{\lambda}\right| d S=\lambda^{2} \int_{M}\left|\varphi_{\lambda}\right| d V
$$

More generally, for any $f \in C^{2}(M)$,

$$
\int_{M}\left(\left(\Delta+\lambda^{2}\right) f\right)\left|\varphi_{\lambda}\right| d V=2 \int_{\mathcal{N}_{\varphi_{\lambda}}} f\left|\nabla \varphi_{\lambda}\right| d S
$$

To obtain lower bounds on $\mathcal{H}^{n-1}\left(\mathcal{N}_{\varphi_{\lambda}}\right)$, we need lower bounds on $\left\|\varphi_{\lambda}\right\|_{L^{1}}$ and upper bounds on $\left|\nabla \varphi_{\lambda}\right|$. The following lower bound is new:

Proposition 2. For any $(M, g)$ and any $L^{2}$-normalized eigenfunction, $\left\|\varphi_{\lambda}\right\|_{L^{1}} \geq$ $C_{g} \lambda^{-\frac{n-1}{4}}$.

Combining Propositions 1 and 2 (and applying the Schwartz inequality to $\left\|\varphi_{\lambda}\right\| \|_{L^{1}}$ ), we obtain

Corollary 2. For any $C^{\infty}$ Riemannian manifold, there exists constants $C, c>0$ so that

$$
C \lambda^{2-\frac{n-1}{4}} \leq C \lambda^{2}\left\|\varphi_{\lambda}\right\|_{L^{1}}=\int_{\mathcal{N}_{\varphi_{\lambda}}}\left|\nabla \varphi_{\lambda}\right| d S \leq c \lambda^{2} \operatorname{Vol}(M)^{1 / 2}
$$

The upper bound is sharp (in terms of order of magnitude) and is achieved by the plane wave eigenfunctions on flat tori and by highest weight spherical harmonics (see $\S 2)$.

We then further use the local Weyl law bound $\left\|\nabla \varphi_{\lambda}\right\|_{C^{0}} \leq C \lambda^{\frac{n+1}{2}}$, again valid on any $n$-dimensional Riemannian manifold, to obtain

Lemma 1. For any $C^{\infty}$ Riemannian manifold, there exists constants $C, c>0$ so that

$$
\int_{\mathcal{N}_{\varphi_{\lambda}}}\left|\nabla \varphi_{\lambda}\right| d S \leq\left(\sup _{x \in \mathcal{N}_{\varphi_{\lambda}}}\left|\nabla \varphi_{\lambda}(x)\right|\right) \mathcal{H}^{n-1}\left(\mathcal{N}_{\varphi_{\lambda}}\right) \leq C \lambda^{\frac{n+1}{2}} \mathcal{H}^{n-1}\left(\mathcal{N}_{\varphi_{\lambda}}\right)
$$

Theorem 1 follows by combining Corollary 2 and Lemma 1 and by dividing both sides by $\lambda^{\frac{n+1}{2}}$.

In $\S 3$, we discuss possible improvements of the lower bound.

The identity for general $f \in C^{2}(M)$ of Proposition 1 can be used to investigate the equidistribution of nodal sets equipped with the surface measure $\lambda^{-2}\left|\nabla \varphi_{\lambda}\right| d S$. Various results of this kind are given in [Z2]. 
0.1. Other level sets. These results generalize easily to any level set $\mathcal{N}_{\varphi_{\lambda}}^{c}:=\left\{\varphi_{\lambda}=\right.$ $c$. Let $\operatorname{sgn}(x)=\frac{x}{|x|}$.

Proposition 3. For any $C^{\infty}$ Riemannian manifold, and any $f \in C(M)$ we have,

$$
\int_{M} f\left(\Delta+\lambda^{2}\right)\left|\varphi_{\lambda}-c\right| d V+\lambda^{2} c \int f \operatorname{sgn}\left(\varphi_{\lambda}-c\right) d V=2 \int_{\mathcal{N}_{\varphi_{\lambda}^{c}}^{c}} f\left|\nabla \varphi_{\lambda}\right| d S
$$

This identity has similar implications for $\mathcal{H}^{n-1}\left(\mathcal{N}_{\varphi_{\lambda}}^{c}\right)$ and for the equidistribution of level sets. Note that if $c>\sup \left|\varphi_{\lambda}(x)\right|$ then indeed both sides are zero.

Corollary 3. For $c \in \mathbb{R}_{+}$

$$
\lambda^{2} \int_{\varphi_{\lambda} \geq c} \varphi_{\lambda} d V=\int_{\mathcal{N}_{\varphi_{\lambda}}^{c}}\left|\nabla \varphi_{\lambda}\right| d S \leq \lambda^{2} \operatorname{Vol}(M)^{1 / 2} .
$$

Consequently, if $c>0$

$$
\mathcal{H}^{n-1}\left(\mathcal{N}_{\varphi_{\lambda}}^{c}\right)+\mathcal{H}^{n-1}\left(\mathcal{N}_{\varphi_{\lambda}}^{-c}\right) \geq C_{g} \lambda^{2-\frac{n+1}{2}} \int_{\left|\varphi_{\lambda}\right| \geq c}\left|\varphi_{\lambda}\right| d V
$$

Of course, $\int_{\left|\varphi_{\lambda}\right| \geq c}\left|\varphi_{\lambda}\right| d V \leq \| \varphi_{\lambda}||_{L^{1}}$, so the lower bound for the $c$-level sets cannot be better than the lower bound for nodal sets. Since $\int_{\left|\varphi_{\lambda}\right| \geq c}\left|\varphi_{\lambda}\right| d V \geq\left\|\varphi_{\lambda}\right\|_{L^{1}}-$ $c V o l\left\{\left(\left|\varphi_{\lambda}\right| \geq c\right\}\right.$, in the cases where $\left\|\varphi_{\lambda}\right\|_{L^{1}} \geq \epsilon_{0}>0$, this lower bound on $\mathcal{H}^{n-1}\left(\mathcal{N}_{\varphi_{\lambda}}^{c} \cup\right.$ $\left.\mathcal{N}_{\varphi_{\lambda}}^{-c}\right)$ is comparable to that for nodal sets when $c<\epsilon_{0}$.

We observe that by the co-area formula,

$$
\lambda^{2}=\int_{M}\left|\nabla \varphi_{\lambda}\right|^{2} d V=\int_{\min \left\{\varphi_{\lambda}\right\}}^{\max \left\{\varphi_{\lambda}\right\}}\left(\int_{\mathcal{N}_{\varphi_{\lambda}}^{c}}\left|\nabla \varphi_{\lambda}\right| d S\right) d c .
$$

The bounds on the nodal set measure the extent to which 0 is an "exceptional value" of $\varphi_{\lambda}$.

Finally, we should explain the connection of our results to those of R. T. Dong. The identity (4) is closely related to the one in [D], Theorem 2.1: Let $q_{\lambda}(x)=$ $\left|\nabla \varphi_{\lambda}(x)\right|^{2}+\frac{\lambda^{2} \varphi_{\lambda}^{2}}{n}$. Then for any domain $\Omega \subset M$ with smooth boundary,

$$
\mathcal{H}^{n-1}\left(\mathcal{N}_{\varphi_{\lambda}} \cap \Omega\right)=\frac{1}{2} \int_{\Omega} \frac{\left(\Delta+\lambda^{2}\right)\left|\varphi_{\lambda}\right|}{\sqrt{q_{\lambda}}} d V
$$

where $d V$ is the volume form of $g$. More precisely, let $\mathcal{T}_{\epsilon}$ denote the $\epsilon$-tube around the singular set $\Sigma\left(\varphi_{\lambda}\right)=\left\{x \in \mathcal{N}_{\varphi_{\lambda}}: \nabla \varphi_{\lambda}(x)=0\right\}$, and define the integral by

$$
\lim _{\epsilon \rightarrow 0} \frac{1}{2} \int_{\Omega \backslash \mathcal{T}_{\epsilon}} \frac{\left(\Delta+\lambda^{2}\right)\left|\varphi_{\lambda}\right|}{\sqrt{q_{\lambda}}} d V
$$

Dong's formula shows that $\frac{\left(\Delta+\lambda^{2}\right)\left|\varphi_{\lambda}\right|}{\sqrt{q_{\lambda}}} d V$ is the codimension one Hausdorff measure $\mathcal{H}^{n-1}$ on $\mathcal{N}_{\varphi_{\lambda}}$. Dong used this identity to obtain an upper bound on $\mathcal{H}^{1}\left(\mathcal{N}_{\varphi_{\lambda}}\right)$ on surfaces. We are using a simpler version where one does not divide by $q_{\lambda}$ to prove a lower bound.

After the second author presented these results at Johns Hopkins, we learned that similar results were obtained independently by Colding and Minicozzi $[\mathrm{CM}]$. Using 
different methods they obtained the sharper lower bound of $\lambda^{\frac{3-n}{4}}$ for $\mathcal{H}^{n-1}\left(\mathcal{N}_{\varphi_{\lambda}}\right)$. Just as we made the final revision to this article, D. Mangoubi sent the authors a preprint in which derives the lower bound $\lambda^{3-n-\frac{1}{n}}$. He also compares the methods of the present article and of [CM, M].

We would like to thank D. Mangoubi, Q. Han and W. Minicozzi for comments on earlier versions of the article. In particular, we thank W. Minicozzi for his helpful comments on the exposition.

\section{Proof of Proposition 1}

We first recall (see [H, HHL, Ch, HHON]) that the singular set

$$
\Sigma\left(\varphi_{\lambda}\right)=\left\{x \in \mathcal{N}_{\varphi_{\lambda}}: \nabla \varphi_{\lambda}(x)=0\right\}
$$

satisfies $\mathcal{H}^{n-2}\left(\Sigma\left(\varphi_{\lambda}\right)\right)<\infty$. Thus, outside of a codimension one subset, $\mathcal{N}_{\varphi_{\lambda}}$ is a smooth manifold, and the Riemannian surface measure $d S=\iota_{\frac{\nabla \varphi_{\lambda}}{\left|\nabla \varphi_{\lambda}\right|}} d V_{g}$ on $\mathcal{N}_{\varphi_{\lambda}}$ is well-defined.

We note that the delta-function on $\mathcal{N}_{\varphi_{\lambda}}$ is the Leray form $\delta\left(\varphi_{\lambda}\right)=\varphi_{\lambda}^{*} \delta_{0}$, i.e. the surface measure $\frac{d V o l}{d \varphi_{\lambda}}=\frac{d S}{\left|\nabla \varphi_{\lambda}\right|}$. The measure

$$
\int f d \mu_{\lambda}:=\int_{M} f\left(\Delta+\lambda^{2}\right)\left|\varphi_{\lambda}\right| d V
$$

can thus be expressed as $\left|\nabla \varphi_{\lambda}\right| d S=\left|\nabla \varphi_{\lambda}\right|^{2} \delta\left(\varphi_{\lambda}\right)$.

Let us now give the proof of Proposition 1. Clearly the second identity implies the first one, and so we need to verify that we have

$$
\int_{M} f d \mu_{\lambda}=2 \int_{\mathcal{N}_{\varphi_{\lambda}}} f\left|\nabla \varphi_{\lambda}\right| d S
$$

We give two (slightly different) proofs.

Proof. Since $d \mu_{\lambda}:=\left(\Delta+\lambda^{2}\right)\left|\varphi_{\lambda}\right| d V=0$ away from $\left\{\varphi_{\lambda}=0\right\}$ it is clear that this distribution is supported on $\left\{\varphi_{\lambda}=0\right\}$. We let $f \in C^{2}(M)$ and consider

$$
\int_{M} f\left(\Delta+\lambda^{2}\right)\left|\varphi_{\lambda}\right| d V=\int_{\left|\varphi_{\lambda}\right| \leq \delta} f\left(\Delta+\lambda^{2}\right)\left|\varphi_{\lambda}\right| d V
$$

Almost all $\delta$ are regular values of $\varphi_{\lambda}$ by Sard's theorem and so we can apply Green's theorem to such values, to obtain

$\int_{\left|\varphi_{\lambda}\right| \leq \delta} f\left(\Delta+\lambda^{2}\right)\left|\varphi_{\lambda}\right| d V-\int_{\left|\varphi_{\lambda}\right| \leq \delta}\left|\varphi_{\lambda}\right|\left(\Delta+\lambda^{2}\right) f d V=\int_{\left|\varphi_{\lambda}\right|=\delta}\left(f \partial_{\nu}\left|\varphi_{\lambda}\right|-\left|\varphi_{\lambda}\right| \partial_{\nu} f\right) d S$.

Here, $\nu$ is the outer unit normal and $\partial_{\nu}$ is the associated directional derivative. For $\delta>0$, we have

$$
\nu=\frac{\nabla \varphi_{\lambda}}{\left|\nabla \varphi_{\lambda}\right|} \text { on }\left\{\varphi_{\lambda}=\delta\right\}, \quad \nu=-\frac{\nabla \varphi_{\lambda}}{\left|\nabla \varphi_{\lambda}\right|} \text { on }\left\{\varphi_{\lambda}=-\delta\right\} .
$$

Letting $\delta \rightarrow 0$ (through the sequence of regular values) we get

$$
\int_{M} f\left(\Delta+\lambda^{2}\right)\left|\varphi_{\lambda}\right| d V=\lim _{\delta \rightarrow 0} \int_{\left|\varphi_{\lambda}\right| \leq \delta} f\left(\Delta+\lambda^{2}\right)\left|\varphi_{\lambda}\right| d V=\lim _{\delta \rightarrow 0} \int_{\left|\varphi_{\lambda}\right|=\delta} f \partial_{\nu}\left|\varphi_{\lambda}\right| d S .
$$


Since $\left|\varphi_{\lambda}\right|= \pm \varphi_{\lambda}$ on $\left\{\varphi_{\lambda}= \pm \delta\right\}$ and by (7), we see that

$$
\begin{aligned}
\int_{M} f\left(\Delta+\lambda^{2}\right)\left|\varphi_{\lambda}\right| d V & =\lim _{\delta \rightarrow 0} \int_{\left|\varphi_{\lambda}\right|=\delta} f \frac{\nabla\left|\varphi_{\lambda}\right|}{|\nabla| \varphi_{\lambda}||} \cdot \nabla\left|\varphi_{\lambda}\right| d S \\
& =\lim _{\delta \rightarrow 0} \sum_{ \pm} \int_{\varphi_{\lambda}= \pm \delta} f\left|\nabla \varphi_{\lambda}\right| d S \\
& =2 \int_{\mathcal{N}_{\varphi_{\lambda}}} f\left|\nabla \varphi_{\lambda}\right| d S .
\end{aligned}
$$

To justify the limit formula, we apply the Gauss-Green formula of geometric measure theory,

$$
\int_{\mathcal{A}(0, \delta)} \operatorname{div} F d y=-\int_{\partial^{*} \mathcal{A}(0, \delta)} F(y) \cdot \nu(y) d \mathcal{H}^{N-1}(y)
$$

to the "annulus" $\mathcal{A}(0, \delta)=\left\{0 \leq \varphi_{\lambda} \leq \delta\right\}$. Here, $\partial^{*} \mathcal{A}_{0, \delta}$ is the "essential boundary" of $\mathcal{A}_{0, \delta}$ (the boundary in the sense of measure theory). In our case, the full boundary is essential, and $\partial^{*} \mathcal{A}(0, \delta)=\mathcal{N}_{\varphi_{\lambda}} \cup \mathcal{N}_{\varphi_{\lambda}}^{\delta}$. Also, $\nu$ is the unit normal, which in our case is $\nu=-\frac{\nabla \varphi_{\lambda}}{\left|\nabla \varphi_{\lambda}\right|}$. To obtain the desired surface integral, we need to set $F=f \nabla \varphi_{\lambda}$, which is a smooth vector field. We obtain

$$
\left.\int_{\mathcal{N}_{\varphi_{\lambda}}} f\left|\nabla \varphi_{\lambda}\right| d S-\int_{\mathcal{N}_{\varphi_{\lambda}}^{\delta}} f\left|\nabla \varphi_{\lambda}\right| d S=\int_{\mathcal{A}(0, \delta)}\left(\nabla f \cdot \nabla \varphi_{\lambda}\right)-\lambda^{2} \varphi_{\lambda}\right) d V=O(\delta) .
$$

The Gauss-Green formula (8) was proved by De Giorgi and Federer under the assumption that $\mathcal{H}^{n-1}\left(\partial^{*} \mathcal{A}(0, \delta)\right)<\infty$, which holds for level sets of eigenfunctions of $C^{\infty}$ metrics since $\mathcal{H}^{n-1}\left(\Sigma\left(\varphi_{\lambda}\right)\right)=0$ as $\mathcal{H}^{n-2}\left(\Sigma\left(\varphi_{\lambda}\right)\right)<\infty$. We refer to Federer $[\mathrm{F}]$ (Sect 2.10.6, page 173 and to Theorem 4.5.11 p. 506.) We also refer to Theorem 1 on p. 209 of $[\mathrm{EG}]$ and $[\mathrm{P}]$ for further discussion.

We now give a second proof:

Proof. We first note that we can express $M$ as the disjoint union

$$
M=\bigcup_{j=1}^{N_{+}(\lambda)} D_{j}^{+} \cup \bigcup_{k=1}^{N_{-}(\lambda)} D_{k}^{-} \cup \mathcal{N}_{\lambda},
$$

where the $D_{j}^{+}$and $D_{k}^{-}$are the positive and negative nodal domains of $\varphi_{\lambda}$, i.e, the connected components of the sets $\left\{\varphi_{\lambda}>0\right\}$ and $\left\{\varphi_{\lambda}<0\right\}$.

Let us assume for the moment that 0 is a regular value for $\varphi_{\lambda}$, i.e., $\Sigma=\emptyset$. Then each $D_{j}^{+}$has smooth boundary $\partial D_{j}^{+}$, and so if $\partial_{\nu}$ is the Riemann outward normal derivative on this set, by the Gauss-Green formula we have

$$
\begin{aligned}
\int_{D_{j}^{+}}\left(\left(\Delta+\lambda^{2}\right) f\right)\left|\varphi_{\lambda}\right| d V & =\int_{D_{j}^{+}}\left(\left(\Delta+\lambda^{2}\right) f\right) \varphi_{\lambda} d V \\
& =\int_{D_{j}^{+}} f\left(\Delta+\lambda^{2}\right) \varphi_{\lambda} d V-\int_{\partial D_{j}^{+}} f \partial_{\nu} \varphi_{\lambda} d S \\
& =\int_{\partial D_{j}^{+}} f\left|\nabla \varphi_{\lambda}\right| d S,
\end{aligned}
$$


using in the last step that $\varphi_{\lambda}$ has eigenvalue $\lambda^{2}$, and that $-\partial_{\nu} \varphi_{\lambda}=\left|\nabla \varphi_{\lambda}\right|$ since $\varphi_{\lambda}=0$ on $\partial D_{j}^{+}$and $\varphi_{\lambda}$ decreases as it crosses $\partial D_{j}^{+}$from $D_{j}^{+}$. A similar argument shows that

$\int_{D_{k}^{-}}\left(\left(\Delta+\lambda^{2}\right) f\right)\left|\varphi_{\lambda}\right| d V=-\int_{D_{k}^{-}}\left(\left(\Delta+\lambda^{2}\right) f\right) \varphi_{\lambda} d V=\int f \partial_{\nu} \varphi_{\lambda} d S=\int_{\partial D_{k}^{-}} f\left|\nabla \varphi_{\lambda}\right| d S$,

using in the last step that $\varphi_{\lambda}$ increases as it crosses $\partial D_{k}^{-}$from $D_{k}^{-}$. If we sum these two identities over $j$ and $k$, we get

$$
\begin{gathered}
\int_{M}\left(\left(\Delta+\lambda^{2}\right) f\right)\left|\varphi_{\lambda}\right| d V=\sum_{j} \int_{D_{j}^{+}}\left(\left(\Delta+\lambda^{2}\right) f\right)\left|\varphi_{\lambda}\right| d V+\sum_{k} \int_{D_{k}^{-}}\left(\left(\Delta+\lambda^{2}\right) f\right)\left|\varphi_{\lambda}\right| d V \\
=\sum_{j} \int_{\partial D_{j}^{+}} f\left|\nabla \varphi_{\lambda}\right| d S+\sum_{k} \int_{\partial D_{k}^{-}} f\left|\nabla \varphi_{\lambda}\right| d S=2 \int_{\mathcal{N}_{\lambda}} f\left|\nabla \varphi_{\lambda}\right| d S
\end{gathered}
$$

using the fact that $\mathcal{N}_{\lambda}$ is the disjoint union of the $\partial D_{j}^{+}$and of the $\partial D_{k}^{-}$.

If 0 is not a regular value of $\varphi_{\lambda}$ we use the Gauss-Green formula for domains with rough boundaries. The preceding argument yields

$$
\int_{D_{j}^{+}}\left(\left(\Delta+\lambda^{2}\right) f\right)\left|\nabla \varphi_{\lambda}\right| d V=\int_{\partial D_{j}^{+}} f\left|\nabla \varphi_{\lambda}\right| d S
$$

and a similar identity for the negative nodal domains. Since $\mathcal{N}_{\lambda} \backslash \Sigma$ is the disjoint union of each of the $\partial D_{j}^{+}$and the $\partial D_{k}^{-}$, we conclude that the same equation holds even when 0 is not a regular value of $\varphi_{\lambda}$.

The attractive feature of this formula is that it immediately gives rather strong results on the integrals with respect to $\left|\nabla \varphi_{\lambda}\right| d S$ over the nodal sets.

LEMMA 1.1. For $f \in C^{2}(M)$, we have $2 \int_{\mathcal{N}_{\varphi_{\lambda}}} f\left|\nabla \varphi_{\lambda}\right| d S=\lambda^{2} \int_{M} f\left|\varphi_{\lambda}\right| d V+O(1)$,

Proof. Our main identity (4) gives, for any test function $f$,

$$
2 \int_{\mathcal{N}_{\varphi_{\lambda}}} f\left|\nabla \varphi_{\lambda}\right| d S=\int_{M}\left|\varphi_{\lambda}\right|\left(\lambda^{2}+\Delta\right) f d V=\lambda^{2} \int f\left|\varphi_{\lambda}\right| d V+O(1),
$$

by the Schwartz inequality and the fact that $\left\|\varphi_{\lambda}\right\|_{L^{2}}=1$.

1.1. Proof of Proposition 2. We now prove Proposition 2:

Proof. Fix a function $\rho \in \mathcal{S}(\mathbb{R})$ having the properties that $\rho(0)=1$ and $\hat{\rho}(t)=0$ if $t \notin[\delta / 2, \delta]$, where $\delta>0$ is smaller than the injectivity radius of $(M, g)$. If we then set

$$
T_{\lambda} f=\rho(\sqrt{-\Delta}-\lambda) f,
$$

we have that $T_{\lambda} \varphi_{\lambda}=\varphi_{\lambda}$. Also, by Lemma 5.1.3 in [So2], $T_{\lambda}$ is an oscillatory integral operator of the form

$$
T_{\lambda} f(x)=\lambda^{\frac{n-1}{2}} \int_{M} e^{i \lambda r(x, y)} a_{\lambda}(x, y) f(y) d y
$$

with $\left|\partial_{x, y}^{\alpha} a_{\lambda}(x, y)\right| \leq C_{\alpha}$. Consequently, $\left\|T_{\lambda} \varphi_{\lambda}\right\|_{L^{\infty}} \leq C \lambda^{\frac{n-1}{2}}\left\|\varphi_{\lambda}\right\|_{L^{1}}$, with $C$ independent of $\lambda$, and so

$$
1=\left\|\varphi_{\lambda}\right\|_{L^{2}}^{2}=\left\langle T \varphi_{\lambda}, \varphi_{\lambda}\right\rangle \leq\left\|T \varphi_{\lambda}\right\|_{L^{\infty}}\left\|\varphi_{\lambda}\right\|_{L^{1}} \leq C \lambda^{\frac{n-1}{2}}\left\|\varphi_{\lambda}\right\|_{L^{1}}^{2} .
$$


We can give another proof based on eigenfunction estimates in [So3], which say that

$$
\left\|\varphi_{\lambda}\right\|_{L^{p}} \leq C \lambda^{\frac{(n-1)(p-2)}{4 p}}, \quad 2<p \leq \frac{2(n+1)}{n-1} .
$$

If we pick such a $2<p<\frac{2(n+1)}{n-1}$, then by Hölder's inequality, we have

$1=\left\|\varphi_{\lambda}\right\|_{L^{2}}^{1 / \theta} \leq\left\|\varphi_{\lambda}\right\|_{L^{1}}\left\|\varphi_{\lambda}\right\|_{L^{p}}^{\frac{1}{\theta}-1} \leq\left\|\varphi_{\lambda}\right\|_{L^{1}}\left(C \lambda^{\frac{(n-1)(p-2)}{4 p}}\right)^{\frac{1}{\theta}-1}$,

$\theta=\frac{p}{p-1}\left(\frac{1}{2}-\frac{1}{p}\right)=\frac{(p-2)}{2(p-1)}$,

which implies $\left\|\varphi_{\lambda}\right\|_{L^{1}} \geq c \lambda^{-\frac{n-1}{4}}$, since $\left(1-\frac{1}{\theta}\right) \frac{(n-1)(p-2)}{4 p}=\frac{n-1}{4}$.

We remark that this lowerbound for $\left\|\varphi_{\lambda}\right\|_{L^{1}}$ is sharp on the standard sphere, since $L^{2}$-normalized highest weight spherical harmonics of degree $k$ with eigenvalue $\lambda^{2}=$ $k(k+n-1)$ have $L^{1}$-norms which are bounded above and below by $k^{(n-1) / 4}$ as $k \rightarrow \infty$. Similarly, the $L^{p}$-upperbounds that we used in the second proof of this $L^{1}$-lowerbound is also sharp because of these functions.

1.2. Lower bounds on $\mathcal{H}^{n-1}\left(\mathcal{N}_{\varphi_{\lambda}}\right)$. We now complete the proof of Theorem 1 along the lines sketched in the introduction:

Proof. Corollary 2 follows from Propositions 1 and 2. It thus remains to prove Lemma 1.

We require the following standard bounds

$$
\left\|\nabla \varphi_{\lambda}\right\|_{C^{0}} \leq C \lambda^{1+\frac{n-1}{2}} .
$$

The proof follows from the local Weyl law,

$$
\sum_{j: \lambda_{j} \leq \lambda}\left|\nabla \varphi_{\lambda}(x)\right|^{2}=C_{n} \lambda^{n+2}+R(x, \lambda),
$$

where $R(x, \lambda)=O\left(\lambda^{n+1}\right)$. See, e.g., Proposition 2.3 of [Z]. It follows that $\left|\nabla \varphi_{\lambda}(x)\right|^{2} \leq$ $|R(x, \lambda)|=O\left(\lambda^{n+1}\right)$. Hence,

$$
\int_{\mathcal{N}_{\varphi_{\lambda}}}\left|\nabla \varphi_{\lambda}\right| d S \leq \lambda^{\frac{n+1}{2}} \int_{\mathcal{N}_{\varphi_{\lambda}}} d S .
$$

We then divide by $\lambda^{\frac{n+1}{2}}$, use the identity of Proposition 1 and then use the lower bound of Proposition 2 to complete the proof of Theorem 1.

1.3. General level sets. The proof of Proposition 3 for general level sets is similar to that for $c=0$, so we will be brief.

LEMMA 1.2. Suppose that $\left\{\varphi_{\lambda}=c\right\}$ is a level set. Then (5) holds, i.e. we have

$$
\left(\Delta+\lambda^{2}\right)\left|\varphi_{\lambda}-c\right| d V+\lambda^{2} c \operatorname{sgn}\left(\varphi_{\lambda}-c\right)=\left|\nabla \varphi_{\lambda}\right|^{2} \delta\left(\varphi_{\lambda}-c\right) \text {. }
$$


Proof. When $\varphi_{\lambda}(x) \neq c$, we have

$$
\left(\Delta+\lambda^{2}\right)\left|\varphi_{\lambda}-c\right| d V=-\operatorname{sgn}\left(\varphi_{\lambda}-c\right) \lambda^{2} c d V .
$$

Hence the difference of the two sides is supported on $\mathcal{N}_{\varphi_{\lambda}}^{c}$. We then repeat the calculation in Proposition 1 with the sets $\left|\varphi_{\lambda}-c\right| \leq \delta$, to get

$\int_{M} f\left(\Delta+\lambda^{2}\right)\left|\varphi_{\lambda}-c\right| d V=$

$-\int_{\left|\varphi_{\lambda}-c\right| \geq \delta} f\left(\operatorname{sgn}\left(\varphi_{\lambda}-c\right) \lambda^{2} c\right) d V+\int_{\left|\varphi_{\lambda}-c\right| \leq \delta} f\left(\Delta+\lambda^{2}\right)\left|\varphi_{\lambda}-c\right| d V$.

By Green's theorem,

$\int_{\left|\varphi_{\lambda}-c\right| \leq \delta} f\left(\Delta+\lambda^{2}\right)\left|\varphi_{\lambda}-c\right| d V-\int_{\left|\varphi_{\lambda}-c\right| \leq \delta}\left|\varphi_{\lambda}-c\right|\left(\Delta+\lambda^{2}\right) f d V=$

$\int_{\left|\varphi_{\lambda}-c\right|=\delta}\left(f \partial_{\nu}\left|\varphi_{\lambda}-c\right|-\left|\varphi_{\lambda}-c\right| \partial_{\nu} f\right) d S$.

Letting $\delta \rightarrow 0$ we get

$$
\lim _{\delta \rightarrow 0} \int_{\left|\varphi_{\lambda}-c\right| \leq \delta} f\left(\Delta+\lambda^{2}\right)\left|\varphi_{\lambda}-c\right| d V=\lim _{\delta \rightarrow 0} \int_{\left|\varphi_{\lambda}-c\right|=\delta} f \partial_{\nu}\left|\varphi_{\lambda}-c\right| d S .
$$

We have

$$
\partial_{\nu}=\frac{\nabla\left|\varphi_{\lambda}-c\right|}{|\nabla| \varphi_{\lambda}-c||} \cdot \nabla=\frac{\nabla \varphi_{\lambda}}{\left|\nabla \varphi_{\lambda}\right|} \cdot \nabla, \quad\left(\text { on }\left\{\left|\varphi_{\lambda}-c\right|=\delta\right\}\right)
$$

and as before,

$$
\lim _{\delta \rightarrow 0} \int_{\left|\varphi_{\lambda}-c\right|=\delta} f \partial_{\nu}\left|\varphi_{\lambda}-c\right| d S=2 \int_{\mathcal{N}_{\varphi_{\lambda}^{c}}^{c}} f\left|\nabla \varphi_{\lambda}\right| d S
$$

We now prove Corollary 3 :

Proof. The first statement follows by integrating $\Delta$ by parts, and by using the identity,

$$
\begin{aligned}
\int_{M}\left|\varphi_{\lambda}-c\right|+c \operatorname{sgn}\left(\varphi_{\lambda}-c\right) d V & =\int_{\varphi_{\lambda}>c} \varphi_{\lambda} d V-\int_{\varphi_{\lambda}<c} \varphi_{\lambda} d V \\
& =2 \int_{\varphi_{\lambda}>c} \varphi_{\lambda} d V,
\end{aligned}
$$

since $0=\int_{M} \varphi_{\lambda} d V=\int_{\varphi_{\lambda}>c} \varphi_{\lambda} d V+\int_{\varphi_{\lambda}<c} \varphi_{\lambda} d V$.

Since for $c>0$ we have $\int_{\varphi_{\lambda}>-c} \varphi_{\lambda} d V=-\int_{\varphi_{\lambda}<-c} \varphi_{\lambda} d V=\int_{\varphi_{\lambda}<-c}\left|\varphi_{\lambda}\right| d V$, we also have

$$
\lambda^{2} \int_{\left|\varphi_{\lambda}\right| \geq c}\left|\varphi_{\lambda}\right| d V=\int_{\mathcal{N}_{\varphi_{\lambda}}^{c}}\left|\nabla \varphi_{\lambda}\right| d S+\int_{\mathcal{N}_{\varphi_{\lambda}}^{-c}}\left|\nabla \varphi_{\lambda}\right| d S, \quad c>0
$$

which yields the second part of the Corollary since $\left|\nabla \varphi_{\lambda}\right|=O\left(\lambda^{\frac{n+1}{2}}\right)$. 
1.4. A curious identity. If we set $f=\varphi_{\lambda_{k}}$ in (4), we obtain

LEMMA 1.3. $\left(\lambda_{j}^{2}-\lambda_{k}^{2}\right) \int_{M} \varphi_{\lambda_{k}}\left|\varphi_{\lambda_{j}}\right| d V=2 \int_{\mathcal{N}_{\varphi_{\lambda_{j}}}} \varphi_{\lambda_{k}}\left|\nabla \varphi_{\lambda_{j}}\right| d S$.

COROLlary 1.4. Suppose that $\lambda_{j}$ is a multiple eigenvalue and that $\lambda_{k}=\lambda_{j}$. Then

$$
\int_{\mathcal{N}_{\varphi_{\lambda_{j}}}} \varphi_{\lambda_{k}}\left|\nabla \varphi_{\lambda_{j}}\right| d S=0
$$

For instance, on a circle we may consider the double eigenvalue $-k^{2}$ with eigenfunctions $\cos k x, \sin k x$, where we may verify the formula. Note that the nodal sets need not intersect. More interesting examples include arithmetic flat tori and round spheres have eigenvalues of high multiplicity (see $\S 2$ ).

By a similar calculation we have,

$$
\left(\lambda_{j}^{2}-\lambda_{k}^{2}\right) \int_{M}\left|\varphi_{\lambda_{k}}\right|\left|\varphi_{\lambda_{j}}\right| d V=2 \int_{\mathcal{N}_{\varphi_{\lambda_{j}}}}\left|\varphi_{\lambda_{k}}\right|\left|\nabla \varphi_{\lambda_{j}}\right| d S-2 \int_{\mathcal{N}_{\varphi_{\lambda_{k}}}}\left|\varphi_{\lambda_{j}}\right|\left|\nabla \varphi_{\lambda_{k}}\right| d S,
$$

hence if $\lambda_{j}$ is a multiple eigenvalue and $\lambda_{k}=\lambda_{j}$,

$$
\int_{\mathcal{N}_{\varphi_{\lambda_{j}}}}\left|\varphi_{\lambda_{k}}\right|\left|\nabla \varphi_{\lambda_{j}}\right| d S=\int_{\mathcal{N}_{\varphi_{\lambda_{k}}}}\left|\varphi_{\lambda_{j}}\right|\left|\nabla \varphi_{\lambda_{k}}\right| d S .
$$

The calculation follows from the fact, by Proposition $1,\left(\Delta+\lambda_{j}^{2}\right)\left|\varphi_{\lambda_{j}}\right|$ is the measure $2\left|\nabla \varphi_{\lambda_{j}}\right| d S$ supported on $\mathcal{N}_{\varphi_{\lambda_{j}}}$.

\section{Examples}

\section{(i) Flat tori}

We first consider the eigenfunctions $\varphi_{k}(x)=\sin \langle k, x\rangle\left(k \in \mathbb{Z}^{n}\right)$ on the flat torus $\mathbf{T}=\mathbb{R}^{n} / \mathbb{Z}^{n}$. The zero set consists of the hyperplanes $\langle k, x\rangle=0 \bmod 2 \pi$. Also, $|\nabla \sin \langle k, x\rangle|^{2}=\cos ^{2}\langle k, x\rangle|k|^{2}$. Since $\cos \langle k, x\rangle=1$ when $\sin \langle k, x\rangle=0$ the integral is simply $|k|$ times the surface volume of the nodal set, which is known to be of size $|k|$. So the upper bound of Corollary 2 is achieved in this example. Also, we have $\int_{\mathbf{T}}|\sin \langle k, x\rangle| d x \geq C$. Thus, our method gives the sharp lower bound $\mathcal{H}^{n-1}\left(\mathcal{N}_{\varphi_{\lambda}}\right) \geq$ $C \lambda^{1}$ in this example.

\section{(ii) Zonal spherical harmonics on $S^{2}$}

The spectral decomposition for the Laplacian is the orthogonal sum of the spaces of spherical harmonics of degree $N$,

$$
L^{2}\left(S^{2}\right)=\bigoplus_{N=0}^{\infty} V_{N},\left.\quad \Delta\right|_{V_{N}}=\lambda_{N} I d
$$

The eigenvalues are given by $\lambda_{N}^{S^{2}}=N(N+1)$ and the multiplicities are given by $m_{N}=2 N+1$. A standard basis is given by the (complex valued) spherical harmonics $Y_{m}^{N}$ which transform by $e^{i m \theta}$ under rotations fixing the poles.

We first consider zonal spherical harmonics $Y_{0}^{N}$ on $S^{2}$, which are real-valued and maximize sup norms among $L^{2}$ normalized spherical harmonics. It is well known that 
$Y_{0}^{N}(r)=\sqrt{\frac{(2 N+1)}{2 \pi}} P_{N}(\cos r)$, where $P_{N}$ is the $N$ th Legendre function and the normalizing constant is chosen so that $\left\|Y_{0}^{N}\right\|_{L^{2}\left(S^{2}\right)}=1$, i.e. $4 \pi \int_{0}^{\pi / 2}\left|P_{N}(\cos r)\right|^{2} d v(r)=1$, where $d v(r)=\sin r d r$ is the polar part of the area form. Its $L^{1}$ norm can be derived from the asymptotics of Legendre polynomials in Theorem 8.21.2 of [S],

$$
P_{N}(\cos \theta)=\sqrt{2}(\pi N \sin \theta)^{-\frac{1}{2}} \cos \left(\left(N+\frac{1}{2}\right) \theta-\frac{\pi}{4}\right)+O\left(N^{-3 / 2}\right)
$$

where the remainder is uniform on any interval $\epsilon<\theta<\pi-\epsilon$. We have

$$
\left\|Y_{0}^{N}\right\|_{L^{1}}=4 \pi \sqrt{\frac{(2 N+1)}{2 \pi}} \int_{0}^{\pi / 2}\left|P_{N}(\cos r)\right| d v(r) \sim C_{0}>0,
$$

i.e. the $L^{1}$ norm is asymptotically a positive constant. Hence $\int_{\mathcal{N}_{Y_{0}^{N}}}\left|\nabla Y_{0}^{N}\right| d s \simeq C_{0} N^{2}$. In this example $\left|\nabla Y_{0}^{N}\right|_{L^{\infty}}=N^{\frac{3}{2}}$ saturates the sup norm bound. So the estimate of Lemma 1 produces the lower bound $\mathcal{H}^{n-1}\left(\mathcal{N}_{\varphi_{\lambda}}\right) \geq \lambda^{\frac{1}{2}}$. The accurate lower bound is $\lambda$, as one sees from the rotational invariance and by the fact that $P_{N}$ has $N$ zeros. The defect in the argument is that the bound $\left|\nabla Y_{0}^{N}\right|_{L^{\infty}}=N^{\frac{3}{2}}$ is only obtained on the nodal components near the poles, where each component has length $\simeq \frac{1}{N}$.

\section{Gaussian beams}

A third example is that of real or imaginary parts of highest weight spherical harmonics $Y_{N}^{N}$ or other Gaussian beams along a closed geodesic $\gamma$ (such as exist on equators of convex surfaces of revolution). We refer to $[\mathrm{R}]$ for background. Gaussian beams are Gaussian shaped lumps which are concentrated on $\lambda^{-\frac{1}{2}}$ tubes $\mathcal{T}_{\lambda^{-\frac{1}{2}}}(\gamma)$ around closed geodesics and have height $\lambda^{\frac{n-1}{4}}$. We note that their $L^{1}$ norms decrease like $\lambda^{-\frac{(n-1)}{4}}$, i.e. they saturate Proposition 2. In such cases we have $\int_{\mathcal{N}_{\varphi_{\lambda}}}\left|\nabla \varphi_{\lambda}\right| d S \simeq$ $\lambda^{2}\left\|\varphi_{\lambda}\right\|_{L^{1}} \simeq \lambda^{2-\frac{n-1}{4}}$. However, the gradient bound $\left\|\nabla \varphi_{\lambda}\right\|_{L^{\infty}}=O\left(\lambda^{\frac{n+1}{2}}\right)$ is far from sharp for Gaussian beams, the sharp upper bound being $\lambda^{1+\frac{n-1}{4}}$. If we use these estimates on $\left\|\varphi_{\lambda}\right\|_{L^{1}}$ and $\left\|\nabla \varphi_{\lambda}\right\|_{L^{\infty}}$, our method gives $\mathcal{H}^{n-1}\left(\mathcal{N}_{\varphi_{\lambda}}\right) \geq C \lambda^{1-\frac{n-1}{2}}$, while $\lambda$ is the sharp lower bound for Gaussian beams in the case of surfaces of revolution (or any real analytic case). The defect is again that the gradient estimate is achieved only very close to the closed geodesic of the Gaussian beam. Outside of the tube $\mathcal{T}_{\lambda^{-\frac{1}{2}}}(\gamma)$ of radius $\lambda^{-\frac{1}{2}}$ around the geodesic, the Gaussian beam and all of its derivatives decay like $e^{-\lambda d^{2}}$ where $d$ is the distance to the geodesic. Hence $\int_{\mathcal{N}_{\varphi_{\lambda}}}\left|\nabla \varphi_{\lambda}\right| d S \simeq$ $\int_{\mathcal{N}_{\varphi_{\lambda}} \cap \mathcal{T}{ }_{\lambda}-\frac{1}{2}}(\gamma)\left|\nabla \varphi_{\lambda}\right| d S$. Applying the gradient bound for Gaussian beams to the latter integral gives $\mathcal{H}^{n-1}\left(\mathcal{N}_{\varphi_{\lambda}} \cap \mathcal{T}_{\lambda^{-\frac{1}{2}}}(\gamma)\right) \geq C \lambda^{1-\frac{n-1}{2}}$, which is sharp since the intersection $\mathcal{N}_{\varphi_{\lambda}} \cap \mathcal{T}_{\lambda^{-\frac{1}{2}}}(\gamma)$ cuts across $\gamma$ in $\simeq \lambda$ equally spaced points (as one sees from the Gaussian beam approximation).

\section{Potential improvements}

As mentioned above, Theorem 1 is based on the identity in Proposition 1 together with a lower bound on $\left\|\varphi_{\lambda}\right\|_{L^{1}}$ and an upper bound on $\left|\nabla \varphi_{\lambda}\right|$. Potential improvements could come from modifying any of these three inputs. The weakest link is the sup 
norm estimate on $\left|\nabla \varphi_{\lambda}\right|$. As we have seen in examples, it is rarely achieved anywhere on $M$, and even when it is, it is only achieved on a small portion of $\mathcal{N}_{\varphi_{\lambda}}$.

The first potential improvement is to use test functions $f$ other than $f \equiv 1$ to generate further identities. For instance if $f=\left|\nabla \varphi_{\lambda}\right|^{2}$, then we get the identity

$$
\int_{M}\left|\nabla \varphi_{\lambda}\right|^{2}\left(\Delta+\lambda^{2}\right)\left|\varphi_{\lambda}\right| d V=\int_{\mathcal{N}_{\varphi_{\lambda}}}\left|\nabla \varphi_{\lambda}\right|^{3} d S
$$

Bochner's identity can be used to simplify the left side. In this way one may try to use $L^{p}$ estimates rather than sup norm estimates of $\left|\nabla \varphi_{\lambda}\right|$.

The lower bound of Proposition 2 is sharp among the class of all $(M, g)$ since it is achieved by highest weight spherical harmonics and other Gaussian beams. However, most $(M, g)$ do not have Gaussian beams (which require existence of stable elliptic closed geodesics), and one might hope to improve Proposition 2 on manifolds with special geometries. It would be particularly interesting to determine the $(M, g)$ or the eigenfunction sequences for which $\left\|\varphi_{\lambda}\right\|_{L^{1}} \geq C>0$. For such eigenfunctions, we would have

$$
\text { (i) } C \lambda^{2} \leq \int_{\mathcal{N}_{\varphi_{\lambda}}}\left|\nabla \varphi_{\lambda}\right| d S \leq c \lambda^{2} \operatorname{Vol}(M)^{1 / 2}, \quad \text { (ii) } \mathcal{H}^{n-1}\left(\mathcal{N}_{\varphi_{\lambda}}\right) \geq C \lambda^{2-\frac{n+1}{2}} .
$$

Thus, in such cases, the upper and lower bounds of (i) have the order of magnitude. As we have seen, the usual exponential eigenfunctions on flat tori or for zonal spherical harmonics on the standard sphere satisfy these lower bounds. It seems interesting to ask whether $\left\|\varphi_{\lambda}\right\|_{L^{1}} \geq C$ for eigenfunctions on negatively curved manifolds (for instance). If so, almost the whole sequence of eigenfunctions would satisfy $\left|\varphi_{\lambda_{j}}\right|^{2} \rightarrow 1$ (weak ${ }^{*}$ ) and $\left\|\varphi_{\lambda_{j}}\right\|_{L^{1}} \rightarrow 0$, and that would indicate (roughly speaking) that the eigenfunctions have a more and more equidistributed set of high and narrow peaks separated by low troughs. This does not contradict quantum ergodicity but it may be a rare phenomenon. We refer to [Z2] for further discussion.

We note that minor improvements are also possible using the argument of [SoZ], which shows that the bound above on $\left\|\nabla \varphi_{\lambda}\right\|_{L^{\infty}}$ is rarely obtained anywhere for metrics on $M$, and could be improved by a factor of $\frac{1}{\sqrt{\log \lambda}}$ if $(M, g)$ is negatively curved.

3.1. Estimates on small balls. Another potential source of improvements is to decompose $M$ into small balls or cubes and to used scaled identities on each ball or cube. More precisely, we could use test functions $f=\chi\left(\lambda\left(x-x_{0}\right)\right)$ where $\chi \in C_{0}^{\infty}\left(\mathbb{R}^{n}\right)$ is a smooth cutoff function, equal to one near 0 . We let $D_{\lambda}^{x_{0}}$ denote the local dilation operator $D_{\lambda}^{x_{0}} u\left(x_{0}+y\right):=u\left(x_{0}+\frac{y}{\lambda}\right)$ with respect to some local coordinates. It converts a high frequency eigenfunction into a low frequency eigenfunction. Then $\chi\left(\lambda\left(x-x_{0}\right)\right)=\left(D_{\lambda}^{x_{0}}\right)^{-1} \chi\left(x-x_{0}\right)$, and $D_{\lambda}^{-1} \Delta D_{\lambda} \sim \lambda^{2} \Delta_{0}^{x_{0}}$ (the Euclidean Laplacian with coordinates frozen at $x_{0}$ in the local coordinates). Hence $\Delta \chi\left(\lambda\left(x-x_{0}\right)\right) \simeq$ $\lambda^{2} D_{\lambda}^{x_{0}}\left(\Delta_{0}^{x_{0}} \chi\right)$ and from (4) we have

$$
\int_{\mathcal{N}_{\varphi_{\lambda}} \cap B\left(x_{0}, \frac{C}{\lambda}\right)} \chi\left(\lambda\left(x-x_{0}\right)\right)\left|\nabla \varphi_{\lambda}\right| d S \simeq \lambda^{2} \int_{B\left(x_{0}, \frac{C}{\lambda}\right)}\left(D_{\lambda}^{x_{0}}\left(I+\Delta_{0}^{x_{0}}\right) \chi\right)\left|\varphi_{\lambda}\right| d V .
$$

One can produce similar inequalities on larger scaled balls. We can then cover the manifold with such balls and partition the balls into the class of small balls where $\left|\nabla \varphi_{\lambda}\right|$ is of average size $\lambda$ and those where it is of the much larger size $\lambda^{\frac{n+1}{2}}$ in our 
sup norm estimate. The latter balls are quite rare. However, we leave this for future investigation.

\section{References}

[ACF] H. Alt, L. Caffarelli, and A. Friedman, Variational problems with two phases and their free boundaries. Trans. Amer. Math. Soc. 282 (1984), no. 2, 431-461.

[B] J. Brüning, Über Knoten Eigenfunktionen des Laplace-Beltrami Operators, Math. Z. 158 (1978), 15-21.

[Ch] S. Y. Cheng, Eigenfunctions and nodal sets. Comment. Math. Helv. 51 (1976), no. 1, 43-55.

$[\mathrm{CM}]$ T. Colding and W. P. Minicozzi II, Lower bounds for nodal sets of eigenfunctions, arXiv:1009.4156. Comm. Math. Phys. (to appear)

[D] R. T. Dong, Nodal sets of eigenfunctions on Riemann surfaces. J. Differential Geom. 36 (1992), no. 2, 493-506.

[DF] H. Donnelly and C. Fefferman, Nodal sets of eigenfunctions on Riemannian manifolds, Invent. Math. 93 (1988), 161-183.

[DF2] H. Donnelly and C. Fefferman, Nodal sets for eigenfunctions of the Laplacian on surfaces, J. Amer. Math. Soc. 3(2) (1990), 333-353.

[EG] L.C. Evans and R. Gariepy: Measure theory and fine properties of functions, CRC Press, Boca Raton, Ann Arbor, and London, 1992

[F] H. Federer, Geometric measure theory. Die Grundlehren der mathematischen Wissenschaften, Band 153 Springer-Verlag New York Inc., New York 1969

[H] Q. Han, Singular sets of solutions to elliptic equations. Indiana Univ. Math. J. 43 (1994), no. 3, 983-1002.

[HHL] Q. Han, R. Hardt, an F. H. Lin, Geometric measure of singular sets of elliptic equations. Comm. Pure Appl. Math. 51 (1998), no. 11-12, 1425-1443.

[HL] Q. Han and F. H. Lin, Nodal sets of solutions of Elliptic Differential Equations, book in preparation (online at http://www.nd.edu/ qhan/nodal.pdf).

[HHON] R. Hardt, M. Hoffmann-Ostenhof, T. Hoffmann-Ostenhof and N. Nadirashvili, Critical sets of solutions to elliptic equations. J. Differential Geom. 51 (1999), no. 2, 359-373.

[HS] R. Hardt and L. Simon, Nodal sets for solutions of elliptic equations. J. Differential Geom. 30 (1989), no. 2, 505-522.

[Ho] L. Hörmander, The Analysis of Linear Partial Differential Operators, Grund. Math. Wiss. 256, Springer-Verlag, New York, 1983.

[Lin] F. H. Lin, Nodal sets of solutions of elliptic and parabolic equations. Comm. Pure Appl. Math. 44 (1991), no. 3, 287-308.

[M] D. Mangoubi, A remark on recent lower bounds for nodal sets, (arXiv 1010.4579).

[P] W. F. Pfeffer, The Gauss-Green theorem. Adv. Math. 87 (1991), no. 1, 93-147.

[R] J. Ralston, Gaussian beams and the propagation of singularities. Studies in partial differential equations, 206-248, MAA Stud. Math., 23, Math. Assoc. America, Washington, DC, 1982.

[Sa] A. Savo, Lower bounds for the nodal length of eigenfunctions of the Laplacian. Ann. Global Anal. Geom. 19 (2001), no. 2, 133-151.

[So] C. D. Sogge, Oscillatory integrals and spherical harmonics. Duke Math. J. 53 (1986), no. $1,43-65$.

[So2] C. D. Sogge, Fourier integrals in classical analysis. Cambridge Tracts in Mathematics, 105. Cambridge University Press, Cambridge, 1993.

[So3] C. D. Sogge, Concerning the $L^{p}$ norm of spectral clusters for second-order elliptic operators on compact manifolds. J. Funct. Anal. 77 (1988), no. 1, 123-138.

[SoZ] C. D. Sogge and S. Zelditch, Riemannian manifolds with maximal eigenfunction growth. Duke Math. J. 114 (2002), no. 3, 387-437.

[S] G. Szegö, Orthogonal Polynomials, Amer. Math. Soc. Colloq. Publ., vol. 23, Amer. Math. Soc., Providence, RI, 1991.

[S2] G. Szegö, Inequalities for the zeros of Legendre polynomials and related functions. Trans. Amer. Math. Soc. 39 (1936), no. 1, 1-17. 
[Y] S.T. Yau, Survey on partial differential equations in differential geometry. Seminar on Differential Geometry, pp. 3-71, Ann. of Math. Stud., 102, Princeton Univ. Press, Princeton, N.J., 1982.

[Z] S. Zelditch, Real and complex zeros of Riemannian random waves, Spectral Analysis in Geometry and Number Theory - Motoko Kotani, Tohoku University, and Hisashi Naito and Tatsuya Tate, Nagoya University, Editors - AMS, 2009.

[Z2] S. Zelditch, Quantum ergodicity and equidistribution of real nodal sets (in preparation).

Department of Mathematics, Johns Hopkins University, Baltimore, MD 21218, USA

E-mail address: sogge@jhu.edu

Department of Mathematics, Northwestern University, Evanston IL, 60208-2730, USA

E-mail address: zel@math.jhu.edu 